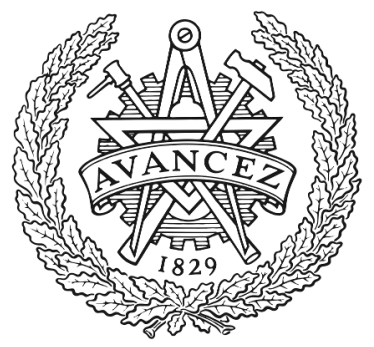

CHALMERS

UNIVERSITY OF TECHNOLOGY

\title{
ToF-SIMS imaging of dual biomolecular monolayer gradients
}

Downloaded from: https://research.chalmers.se, 2023-04-26 11:48 UTC

Citation for the original published paper (version of record):

Evenbratt, H., Munem, M., Malmberg, P. (2020). ToF-SIMS imaging of dual biomolecular monolayer gradients. Biointerphases, 15(6). http://dx.doi.org/10.1116/6.0000621

N.B. When citing this work, cite the original published paper. 


\section{ToF-SIMS imaging of dual biomolecular monolayer gradients}

Cite as: Biointerphases 15, 061014 (2020); https://doi.org/10.1116/6.0000621

Submitted: 11 September 2020 . Accepted: 30 November 2020 . Published Online: 22 December 2020

(iD) Hanne Evenbratt, Marwa Munem, and (D) Per Malmberg

\section{COLLECTIONS}

Paper published as part of the special topic on Special Topic Collection: Biomimetics of Biointerfaces BIOMIM2020
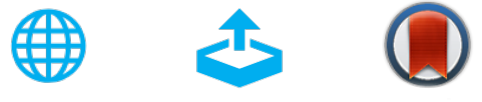

\section{ARTICLES YOU MAY BE INTERESTED IN}

Analyzing 3D hyperspectral TOF-SIMS depth profile data using self-organizing map-relational perspective mapping

Biointerphases 15, 061004 (2020); https://doi.org/10.1116/6.0000614

Replica molding of cicada wings: The role of water at point of synthesis on nanostructure feature size

Biointerphases 15, 061017 (2020); https://doi.org/10.1116/6.0000637

Improved recovery of cryopreserved cell monolayers with a hyaluronic acid surface treatment

Biointerphases 15, 061015 (2020); https://doi.org/10.1116/6.0000613

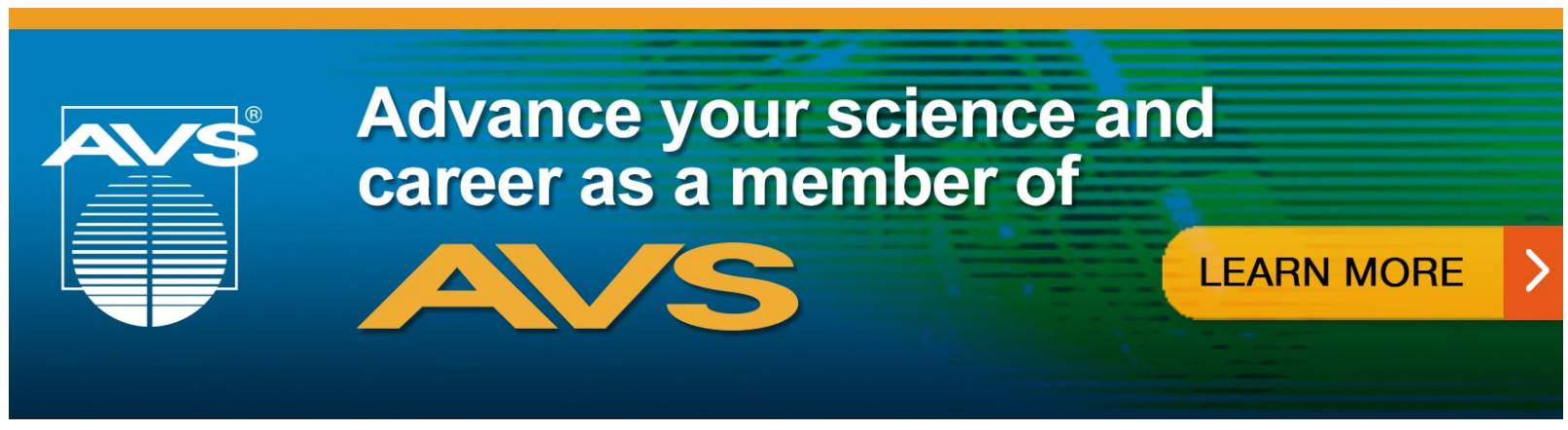




\title{
ToF-SIMS imaging of dual biomolecular monolayer gradients
}

\author{
Cite as: Biointerphases 15, 061014 (2020); doi: $10.1116 / 6.0000621$ \\ Submitted: 11 September 2020 . Accepted: 30 November 2020 . \\ Published Online: 22 December 2020 \\ Hanne Evenbratt, $^{1, a)}$ (D) Marwa Munem, ${ }^{2}$ and Per Malmberg ${ }^{2}$ (D) \\ AFFILIATIONS \\ ${ }^{1}$ Cline Scientific AB, SE-431 53 Mölndal, Sweden \\ ${ }^{2}$ Department of Chemistry and Chemical Engineering, Chalmers University of Technology, SE-472 96 Gothenburg, Sweden
}

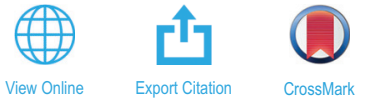

Note: This paper is part of the Biointerphases Special Topic Collection on Biomimetics of Biointerfaces.

${ }^{a}$ Electronic mail: hanne.evenbratt@clinescientific.com

\begin{abstract}
Precise characterization of a monolayer of two different biomolecules in a gradient pattern on a glass surface puts high demand on the method used. Some techniques can detect protein monolayers but not on a glass surface. Others can distinguish between different proteins but not identify a gradient pattern. Here, we used ToF-SIMS to validate the complete surface composition, checking all the necessary boxes. As these types of surfaces can dictate sensitive cell behaviors, the precision on a nanolevel is crucial, and to visualize and determine the molecular distribution become essential. The dual monolayer consisted of laminin 521 and one of three other biomolecules of different sizes, epidermal growth factor, growth differentiation factor 5, or bovine serum albumin, creating opposing gradient patterns. The resulting ToF-SIMS imaging and line scan data provided detailed information on the distribution of the adsorbed proteins.
\end{abstract}

Published under license by AVS. https://doi.org/10.1116/6.0000621

\section{INTRODUCTION}

Surface analysis with nanometer resolution is today viable using different techniques. However, the precise characterization of two biomolecules in a dual gradient pattern on a gold nanoparticle (AuNP) and glass substrate puts high demand on the method used. Depending on the focus and scope, a combination of surface analysis techniques such as time-of-flight secondary ion mass spectrometry (ToF-SIMS), scanning electron microscopy (SEM), x-ray photoelectron spectroscopy (XPS), quartz crystal microbalance with dissipation (QCM-D) monitoring, surface plasmon resonance (SPR), enzyme-linked immunosorbent assay (ELISA), and different microscopic methods could be employed. Due to the nature of the specific nanogradient surfaces used here, many techniques had to be discarded. XPS can detect adsorbed protein amounts, but the distinction between two proteins on the same surface is difficult. Also, the AuNP creates an issue, at least in the densest area of the nanogradient. ${ }^{1,2}$ The key part of the QCM-D technique is that it is a microbalance. The amount of added proteins can be measured as well as the viscoelastic properties of the resulting coverage, etc. ${ }^{3}$ but not the distribution along a gradient. SPR is a commonly used technique to study the kinetics of biomolecule binding and has been used on similar gradients but cannot be used on a glass substrate. ${ }^{4,5}$ Microscopic techniques are easy to use with nanogradient surfaces. However, the AuNPs can create problems in, e.g., fluorescence approaches, ELISA included, where the fluorescent signal is quenched near the AuNPs ${ }^{6}$ or when relying on multiphoton induced luminescence (MIL) where the interparticle distance dictates the presence of a MIL signal.

A suitable candidate technique to use is ToF-SIMS imaging, providing a high spatial resolution of about $400 \mathrm{~nm},{ }^{8}$ low surface impact, and high reliability when used for visualizing the molecular distribution. $^{9-12}$ ToF-SIMS is a well-established state-of-the-art technique for identifying specific elements and enabling detailed studies of amino acid distribution in adsorbed proteins with ppm level of sensitivity. ${ }^{13}$ Compared to other protein distribution imaging methods, e.g., fluorescence microscopy, ToF-SIMS does not need specific probes and or antibodies for protein identification and offers the possibility to distinguish between different biomolecules easier than, e.g., an SPR imaging setup. ${ }^{14}$

The present study aimed to employ ToF-SIMS to visualize the immobilized biomolecules' distribution along the nanoparticle gradient and distinguish between the biomolecules and the surrounding laminin. The nanogradients used here consist of a glass surface with AuNPs attached in a gradient pattern determined using SEM. 
The glass surrounding the nanoparticles was covered with extracellular matrix protein laminin 521, and the nanoparticles were functionalized with one of three different proteins: epidermal growth factor (EGF), growth differentiation factor 5 (GDF5), or bovine serum albumin (BSA) [Figs. 3(b)-3(e) show the basic nanogradient setup]. These biomolecules are different in size $(6.2,26.8,66.5 \mathrm{kDa}$, respectively) and may thus distribute a bit differently along the gradient depending on, e.g., the number of attachment points to the AuNPs (see Figures S7-11). The nanostructured surfaces themselves, their topography, and functionalization, i.e., with precisely distributed biomolecules, have been shown to influence several different events such as cell adhesion, viability, and tissue integration. ${ }^{15-20}$ Thus, when purposefully functionalized, these surfaces can dictate cell responses in, e.g., cell migration and cell differentiation. Therefore, the demand for knowledge of the precise surface composition, on a nanolevel, is evident. ${ }^{21-26}$ The information gained through mainly ToF-SIMS measurements of the gradient surfaces can thus be necessary, e.g., when interpreting the result from stem cell differentiation on the morphogen gradient.

\section{EXPERIMENT}

\section{A. Preparation of nanogradients}

The nanogradients were provided by Cline Scientific (Cline Scientific AB, Sweden), with $10 \mathrm{~nm}$ citrate-covered AuNPs (Cline Scientific $A B$, Sweden) in a gradient pattern on a glass substrate. A proprietary method developed by Cline Scientific for functionalization of the nanogradient surfaces included the following steps: thiolated streptavidin (SH-streptavidin, ProteinMods, USA) was applied to the nanoparticles and incubated at room temperature. Thereafter, superfluous streptavidin was rinsed off with phosphate-buffered saline (PBS) (Amresco, USA). Next, laminin 521 (Biolamina AB, Sweden) was attached to the glass surface between particles by charge-charge interaction (net charge -130, pI 5.92) and incubated at room temperature, whereafter superfluous laminin was rinsed with PBS.

EGF (R\&D Systems, Bio-Techne, MN, USA) and GDF5 (R\&D Systems, Bio-Techne, MN, USA) were biotinylated to facilitate binding with streptavidin. Biotinylation was performed with an excessive amount of biotin (EZ-Link ${ }^{\mathrm{TM}}$ Sulfo-NHS-LC-Biotin, Thermo Fischer Scientific, MA, USA) according to the instructions provided by the supplier. The superfluous biotin was discarded using repeated wash and centrifugation steps. The biotinylation's success was controlled using a HABA test, resulting in 1-5 biotins/ molecule (HABA/avidin Reagent, Sigma-Aldrich, USA). BSA was bought already biotinylated (Thermo Fisher Scientific, MA, USA). Finally, the biotinylated proteins were attached to the streptavidincoated AuNPs and incubated at $4{ }^{\circ} \mathrm{C}$ overnight. As in the previous steps, superfluous proteins were rinsed off with PBS. After functionalization, the surfaces were rinsed with water and dried, awaiting ToF-SIMS measurements.

\section{B. Preparation of protein standards for ToF-SIMS}

EGF (R\&D Systems, Bio-Techne, MN, USA) $5.4 \times 10^{-5} \mathrm{M}$, BSA-biotin (Thermo Fisher Scientific, MA, USA) $4 \times 10^{-8} \mathrm{M}$, GDF5 (R\&D Systems, Bio-Techne, MN, USA) $7 \times 10^{-6} \mathrm{M}$, and laminin 521 (Biolamina $\mathrm{AB}$, Sweden) $1.3 \times 10^{-7}$ were dissolved according to the specifications accompanying each protein from the manufacturer and deposited dropwise on the glass surface.

\section{SEM}

The gradient pattern was confirmed using scanning electron microscopy (SEM) (Zeiss Ultra 55, Germany) [Fig. 3(a)]. Images were acquired in the secondary/backscattered electron mode at an accelerating voltage of $3.00 \mathrm{kV}$ using an In-Lens detector. The nanogradients had a particle density spanning on average from 1 to 2250 particles $/ \mu \mathrm{m}^{2}$, measured using scanning electron microscopy (SEM) in three lines along the gradient, depicted with standard deviations in Fig. 3(a).

\section{ToF-SIMS}

ToF-SIMS imaging was performed using a TOF.SIMS V (ION-TOF, Münster, Germany), with a bismuth liquid metal ion gun as a primary ion source and a $\mathrm{C}_{60}^{+} 10-\mathrm{keV}$ ion source as a sputter source. Data were recorded in positive and negative ion modes, and spectra were acquired using $\mathrm{Bi}_{3}^{++}(50 \mathrm{keV})$ primary ions. All analyses were performed in the so-called high-current bunched mode, with a pulsed primary ion current of $0.34 \mathrm{pA}$ and a maximum ion dose density of $3 \times 10^{11}$ ions $/ \mathrm{cm}^{2}$, which is below the static limit. ${ }^{27}$ Surfacelab 7 software (v. 7.0; ION-TOF) was used for all spectrum and image processing and analysis. The mass spectra were internally calibrated to signals of $[\mathrm{C}]^{-},[\mathrm{CH}]^{-},\left[\mathrm{C}_{2}\right]^{-}$, $\left[\mathrm{C}_{3}\right]^{-}$and $[\mathrm{C}]^{+}\left[\mathrm{CH}_{2}\right]^{+},\left[\mathrm{CH}_{3}\right]^{+}$, and $\left[\mathrm{C}_{5} \mathrm{H}_{12} \mathrm{~N}\right]^{+}$for negative and positive ion modes, respectively. The gradient surfaces were analyzed using the raster stage scan mode to cover areas between $4000 \times 11200 \mu \mathrm{m}^{2}$ and $4000 \times 11600 \mu \mathrm{m}^{2}$. In total, three sets of each functionalized gradient were analyzed in three different areas. One protein standard was analyzed for each protein type. The red/ green/blue (RGB) color overlays are represented in a linear relationship ranging from a signal intensity of zero as black to $100 \%$ color for the maximum ion count for that respective ion.

\section{RESULTS AND DISCUSSION}

\section{A. ToF-SIMS visualizes biomolecular binding}

ToF-SIMS analysis of the protein standards gave characteristic peaks for the proteins used to functionalize the surfaces. A peak search was used to identify peaks based on their relative intensities relative to the other proteins, as well as principal component analysis (data not shown). This information and information from previous publications on ToF-SIMS protein characterization were used to identify peaks from different biomolecules on the surfaces. ${ }^{13,28,29}$ To determine the gradient pattern, ion signals from each protein's characteristic peaks were added together to be overlaid with the sum of ion signals from the laminin 521 background peaks. Spectra from EGF, BSA, GDF5, and laminin 521 can be seen in supplementary material Fig. S1. ${ }^{31}$

ToF-SIMS macroraster images from the functionalized surfaces are shown in Fig. 1 and are represented as overlay images showing the three biomolecules in green and the laminin background in red. Individual macroraster ion images can be seen in supplementary Figs. S2-S4. ${ }^{31}$ A clear separation between the functionalizing biomolecule and the laminin background for both EGF and GDF5 can be seen in Figs. 1(a) and 1(b). The separation between BSA and 
a

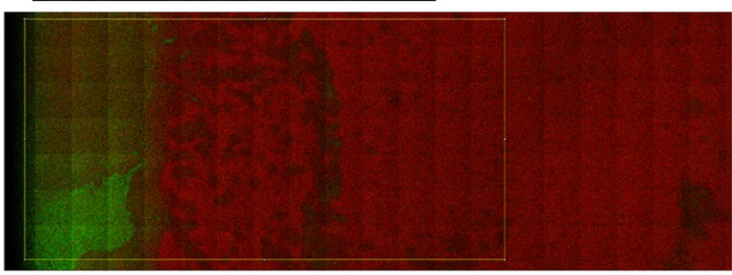

b

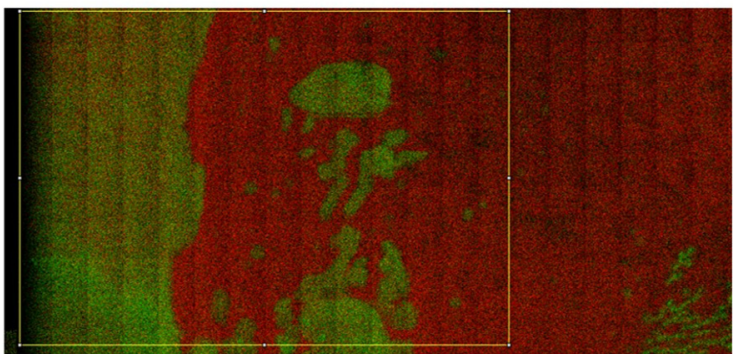

C

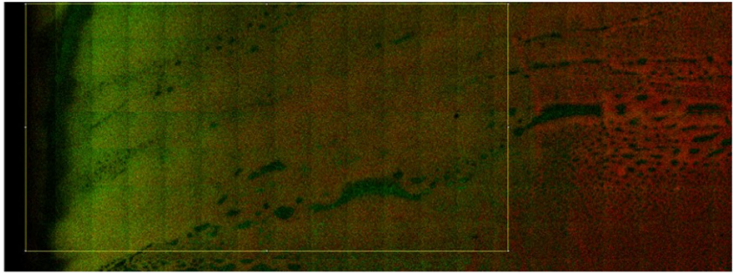

FIG. 1. ToF-SIMS macroraster overlay images showing the functionalized biomolecules EGF (a), GDF (b), and BSA (c) in green contrasted to laminin 521 (red). The yellow rectangles indicate areas used for the line scans, as can be seen in Fig. 2, and cover approximately $3000 \times 4500 \mu \mathrm{m}^{2}$. The wedge at the top of the figure indicates the gradient's direction with the highest particle density on the left side.

laminin in Fig. 1(c) is less clear due to BSA and laminin having several overlapping peaks (see protein references, Figure S1).

The ToF-SIMS surface data can also be represented as line scans showing the gradient distribution of the biomolecules on the surfaces, as shown in Fig. 2. The line scans were based on an average area from the gradient surfaces of approximately 3000 (width) $\times 4500 \mu \mathrm{m}^{2}$ (length). All three biomolecules (laminin excluded) start with a high density on the gradient surface's densest part, followed by descending density to approximately $2500 \mu \mathrm{m}$. The laminin signal shows an opposite behavior, especially for EGF and GDF5, as shown in Figs. 2(a) and 2(b) with a sharply increasing signal at approximately $1500 \mu \mathrm{m}$. For BSA, the laminin signal is at its maximum at around $1000 \mu \mathrm{m}$ with no apparent increase, contrary to the EGF and GDF5 surfaces. Another set of images is shown in supplementary information Fig. S5. ${ }^{31}$ Since the gradient preparation is very consistent between samples, the individual samples analyzed showed very similar results.

\section{B. SEM confirms the gradient pattern}

The ToF-SIMS data show the distribution of molecules along the gradient following EGF, GDF5, and BSA attachment to the a

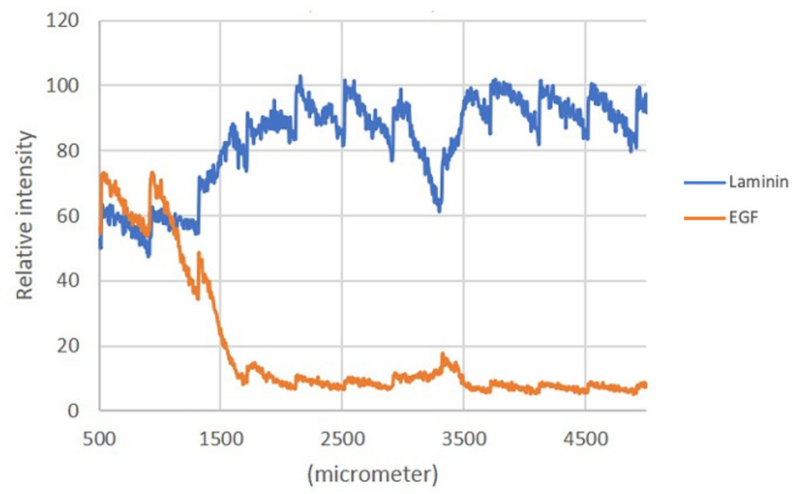

b

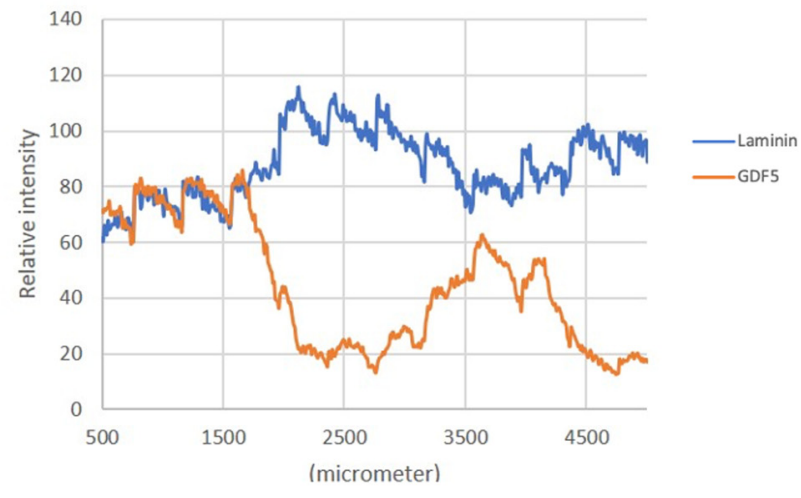

C

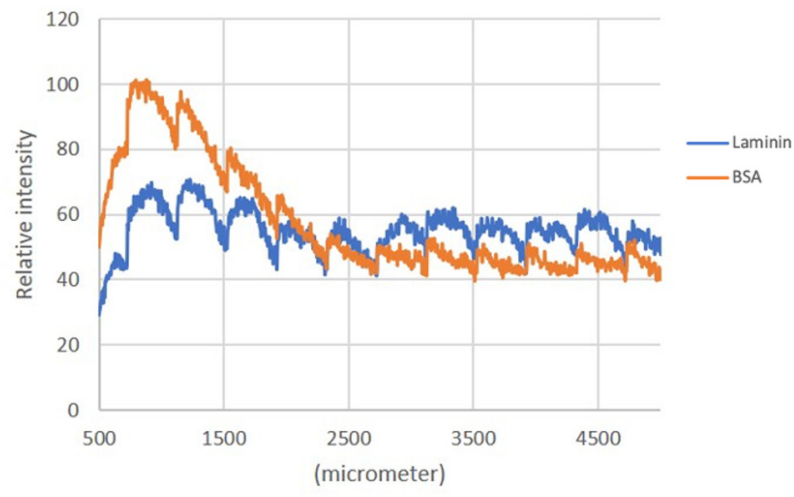

FIG. 2. Line scans of biomolecule distribution based on ToF-SIMS macroraster data showing the distribution of EGF (a), GDF5 (b), and BSA (c) in orange in relationship to laminin in blue. The line scans were made from an area starting approximately $500 \mu \mathrm{m}$ from the edge of the gradient surface (high-density end) and cover a length of ca. $4500 \mu \mathrm{m}$. Line scans were averaged over an area of about $3000 \mu \mathrm{m}$. The relative intensity signals are normalized to the total ion count of the experiment.

AuNPs and laminin on the substrate surrounding the particles. SEM data on AuNP distribution (Fig. 3) confirms a decreasing AuNP density from 500 to $3000 \mu \mathrm{m}$, concurring with the decreasing amounts of biomolecules. The high density of AuNPs and the size of laminin 521 lead to a decreasing amount of laminin toward 

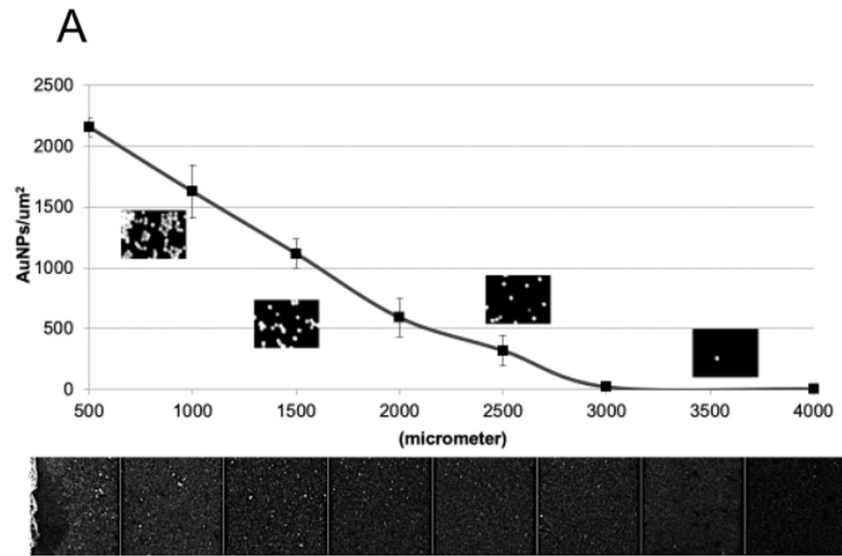

B

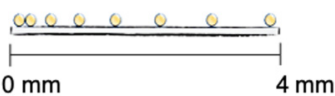

D

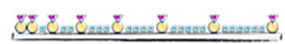

$\mathrm{E}$

C
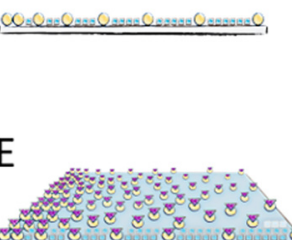

() AuNP

四 Laminin 521

Streptavidin

FIG. 3. AuNP distribution based on SEM measurements shown as the average number of particles per $\mu \mathrm{m}$ (curve) and SEM images along the gradient's length (images). The curve shows the average number of nanoparticles per $\mu \mathrm{m}^{2}$ measured every $500 \mu \mathrm{m}$. The inset is SEM closeup (magnification $\times 200000$ ) of the particles along the gradient. Below the curve, a montage of SEM pictures covering the gradient's length every $500 \mu \mathrm{m}$; each square length is $30 \mu \mathrm{m}$. Illustration (b)-(e) is redrawn based on a figure published in Andreasson et al. (Ref. 18), showing (b) AuNPs (yellow circles) attached in a gradient pattern to a glass substrate. (c) Laminin 521 (blue squares) attached to the glass surface between particles. (d) Streptavidin (purple triangles) attached to AuNPs as a linker to the biotinylated biomolecule. (e) Gradient ready to be functionalized by attaching biomolecules.

the surface's edge (far left Fig. 3, see also Figs. S10 and S11). Only a very weak signal could be detected from the AuNP monitored with ToF-SIMS (see Fig. S6 in the supplementary material), ${ }^{31}$ most likely because the AuNPs are covered by both streptavidin and a biotinylated biomolecule, which hinders the effective production of gold secondary ions.

\section{Discussing differences and usefulness}

As can be seen in Figs. 1(b) and 2(b), the GDF5 gradient has irregularities approximately $3500 \mu \mathrm{m}$ from the edge. These green areas in the middle of red [Fig. 1(b)] and the corresponding additional peak in Fig. 2(b) are due to AuNP aggregates present in the measured area. BSA and laminin 521 in Figs. 1(c) and 2(c) show a slightly different profile than the other two gradient surfaces. It is more difficult to differentiate between these two molecules, but the distinction still shows a clear BSA gradient. The large BSA molecules may, to some extent, cover and thus impede the signal originating from laminin 521 (see Figs. S7, S10, and S11 for molecular sizes and distributional illustration).

Factors such as the density of the AuNP, the number of binding spots, and the biomolecules' size all influence the highest biomolecular binding density possible to achieve as well as the biomolecular gradient slope, further illustrated in Figs. 3 and S7-S11. In this case, the approximately $3000-\mu \mathrm{m}$-long AuNP gradients result in three slightly different profiles depending on the biomolecule attached to the AuNPs. The chemistry used to bind the proteins to their respective parts of the surface, the nanoparticles (biotin-streptavidin), or the surrounding glass (charge-charge interaction) is well known and established. The validation of their respective positions in a dual monolayer gradient on glass (instead of the commonly used gold or silicon surface) has long been challenging. ToF-SIMS is one of the few techniques with the capacity to measure both a monolayer and determine the gradient pattern. ${ }^{17}$

These types of gradient surfaces are of use in, e.g., stem cell differentiation, as models for molecular recognition and interaction in biological systems, cell motility and diagnostics, and practical applications such as cell separation, drug delivery, and sensors in biotechnology, where the molecular composition and control thereof are of the essence. ${ }^{19,20,30}$ Hence, the validation of the surface composition dictating the cell behavior is vital.

\section{SUMMARY AND CONCLUSIONS}

Creating biomolecular monolayers on a glass surface opens up possibilities for precise control over cell reactions combined with the ability to use microscopic techniques to study the results. Here, we use ToF-SIMS to detect biomolecular monolayers with high precision on glass surfaces and more than one bound protein. ToF-SIMS allows for precision measurements of the biomolecule gradients on glass surfaces, which is difficult with other techniques than ToF-SIMS. Moreover, we distinguish between two proteins on each gradient surface and show different binding patterns depending on, e.g., molecular size.

\section{ACKNOWLEDGMENTS}

We thank Associate Professor Reza Mobini, Ph.D. for valuable scientific advice and discussions. This work was performed in part at Chalmers Material Analysis Laboratory, CMAL, and the Infrastructure for Chemical Imaging at Chalmers University of Technology and the University of Gothenburg. This work was also supported by Cline Scientific AB, Sweden, which provided the gradient surfaces. Hanne Evenbratt is employed by and holds stock in Cline Scientific AB. The authors have no additional competing financial interests.

\section{DATA AVAILABILITY}

The data that support the findings of this study are available within the article and its supplementary material. 


\section{REFERENCES}

'E. H. Tronic, Surface Analysis of Adsorbed Proteins: A Multi-Technique Approach to Characterize Surface Structure (University of Washington, Seattle, WA, 2012).

${ }^{\mathbf{2}}$ C. Gruian, E. Vanea, S. Simon, and V. Simon, Biochim. Biophys. Acta Proteins Proteomics 1824, 873 (2012).

${ }^{3}$ F. Höök, B. Kasemo, T. Nylander, C. Fant, K. Sott, and H. Elwing, Anal. Chem. 73, 5796 (2001)

${ }^{4}$ R. Konradi, M. Textor, and E. Reimhult, Biosensors 2, 341 (2012).

${ }^{5}$ A. Lundgren, M. Hulander, J. Brorsson, M. Hermansson, H. Elwing, O. Andersson, B. Liedberg, and M. Berglin, Part. Part. Syst. Charact. 31, 209 (2014). ${ }^{6}$ E. Dulkeith et al., Phys. Rev. Lett. 89, 203002 (2002).

7J. Borglin, S. Guldbrand, H. Evenbratt, V. Kirejev, H. Grönbeck, and M. B. Ericson, Appl. Phys. Lett. 107, 234101 (2015).

${ }^{8}$ Q. P. Vanbellingen, N. Elie, M. J. Eller, S. Della-Negra, D. Touboul, and A. Brunelle, Rapid Commun. Mass Spectrom. 29, 1187 (2015).

${ }^{9}$ P. Kingshott, G. Andersson, S. L. McArthur, and H. J. Griesser, Curr. Opin. Chem. Biol. 15, 667 (2011).

${ }^{10}$ A. M. Belu, D. J. Graham, and D. G. Castner, Biomaterials 24, 3635 (2003).

${ }^{11}$ P. Malmberg, T. Guttenberg, M. B. Ericson, and L. Hagvall, Contact Dermatitis 78, 109 (2018).

${ }^{12}$ P. Malmberg, E. Jennische, D. Nilsson, and H. Nygren, Anal. Bioanal. Chem. 399, 2711 (2011).

${ }^{13}$ B. Li, Y. Ma, S. Wang, and P. M. Moran, Biomaterials 26, 1487 (2005).

${ }^{14}$ R. Michel and D. G. Castner, Surf. Interface Anal. 38, 1386 (2006).
${ }^{15} \mathrm{M}$. Hulander, A. Lundgren, M. Berglin, M. Ohrlander, J. Lausmaa, and H. Elwing, Int. J. Nanomed. 6, 2653 (2011).

${ }^{16}$ A. M. Lipski, C. J. Pino, F. R. Haselton, I.-W. Chen, and V. P. Shastri, Biomaterials 29, 3836 (2008).

${ }^{17} \mathrm{~K}$. Anselme, L. Ploux, and A. Ponche, J. Adhes. Sci. Technol. 24, 831 (2010).

${ }^{18}$ A. Lundgren, Y. Hed, K. Öberg, A. Sellborn, H. Fink, P. Löwenhielm, J. Kelly, M. Malkoch, and M. Berglin, Angew. Chemie Int. Ed. 50, 3450 (2011).

${ }^{19}$ L. Andreasson, H. Evenbratt, and S. Simonsson, Heliyon 6, e04133 (2020).

${ }^{20}$ L. Andreasson, H. Evenbratt, R. Mobini, and S. Simonsson, J. Biotechnol. (published online, 2020).

${ }^{21}$ M. Arnold et al., Nano Lett. 8, 2063 (2008).

${ }^{22}$ R. J. McMurray et al., Nat. Mater. 10, 637 (2011)

${ }^{23}$ M. J. Dalby, N. Gadegaard, R. Tare, A. Andar, M. O. Riehle, P. Herzyk, C. D. W. Wilkinson, and R. O. C. Oreffo, Nat. Mater. 6, 997 (2007).

${ }^{24}$ G. Maheshwari, G. Brown, D. A. Lauffenburger, A. Wells, and L. G. Griffith, J. Cell Sci. 113, 1677 LP (2000).

${ }^{25}$ M. P. Lutolf and J. A. Hubbell, Nat. Biotechnol. 23, 47 (2005).

${ }^{26}$ J. B. Gurdon and P.-Y. Bourillot, Nature 413, 797 (2001).

${ }^{27}$ R. N. S. Sodhi, Analyst 129, 483 (2004).

${ }^{28}$ B. J. Tyler, C. Bruening, S. Rangaranjan, and H. F. Arlinghaus, Biointerphases 6, 135 (2011).

${ }^{\mathbf{2 9}}$ R. N. Foster, E. T. Harrison, and D. G. Castner, Langmuir 32, 3207 (2016).

${ }^{30}$ M. S. Kim, G. Khang, and H. B. Lee, Prog. Polym. Sci. 33, 138 (2008).

${ }^{31}$ See supplementary material at https://doi.org/10.1116/6.0000621 for further information on protein structure, spectra, etc. 